\title{
Food and Nutrition Report
}

\section{Effects of Aqueous Extracts from Grape and Grape Seed on Adenosine Deaminase Activity in Cancerous and Non cancerous Human Gastric and Colon Tissues}

\author{
Zahide Esra Durak ${ }^{1 *}$, Suleyman Buber ${ }^{2}$, Bahadır Ozturk ${ }^{3}$ and Hilmi Kocaoglu ${ }^{4}$ \\ ${ }^{1}$ Ordu University Central Research Laboratory ORDU, Turkey \\ ${ }^{2}$ Ankara University Faculty of Medicine, Dept. of Medical Biochemistry, Ankara, Turkey \\ ${ }^{3}$ Selcuk University Faculty of Medicine, Dept. of Medical Biochemistry, Konya, Turkey \\ ${ }^{4}$ Ankara University Faculty of Medicine, Department of Surgical Oncology, Ankara, Turkey
}

\begin{abstract}
*Corresponding author: Zahide Esra DURAK PhD., Ordu University Central Research Lab, Ordu, Turkey; Email: zaesrad@hotmail.com Article Type: Research, Submission Date: 10 September 2015, Accepted Date: 05 October 2015, Published Date: 10 November 2015.

Citation: Zahide Esra Durak, Suleyman Buber, Bahadır Ozturk and Hilmi Kocaoglu (2015) Effects of Aqueous Extracts from Grape and Grape Seed on Adenosine Deaminase Activity in Cancerous and Non cancerous Human Gastric and Colon Tissues. F Nutr Reprt 1(2): 6-9. doi: https://doi.org/10.24218/fnr.2015.07.
\end{abstract}

Copyright: (c) 2015 Zahide Esra Durak, et al. This is an open-access article distributed under the terms of the Creative Commons Attribution License, which permits unrestricted use, distribution, and reproduction in any medium, provided the original author and source are credited.

\section{Translational Relevance}

Gastric and colon cancers have a big mortality all over the world. Each year, thousands of people die because of these cancers. For some cancer types, agents originated from plants have yielded positive results.

Adenosine deaminase is one of the key enzymes in purine metabolism, the inhibition of which is significant for the therapy and control of cancer process. Therefore, a natural source having potential to inhibit this enzyme is very important.

Our results show that grape constituents can inhibit ADA enzyme significantly. Therefore, results of the present study may be of importance with regard to the cancer therapy and control. We hope this study can lead to further researches on this subject.

\section{Abstract}

\section{Purpose}

We aimed to investigate possible effects of grape and grape seed extracts on adenosine deaminase (ADA) activity in cancerous and non cancerous human gastric and colon tissues to obtain information about possible anticancer properties of grape.

\section{Experimental design}

Cancerous and non cancerous human gastric and colon tissues removed from patients by surgical operations were used in the studies. The extracts were prepared in distilled water. Before and after treatment with the extracts, ADA activities in the samples were measured.

\section{Results}

ADA activity was found to be inhibited by aqueous grape extract in cancerous and non cancerous gastric and colon tissues. ADA activity was found to be lowered by the grape seed extract only in the cancerous gastric tissues but not in the others.

\section{Conclusion}

Our results suggest that inhibition created by the grape constituents might be of importance as to the anticancer potential of grape and grape seed. It is possible that in addition to other proposed mechanisms, accumulated adenosine due to the inhibition of ADA enzyme might play part in the anticancer properties of the grape constituents.

Keywords: Adenosine Deaminase, Grape, Grape Seed, Colon Cancer, Gastric Cancer.

\section{Introduction}

Cancer is one of the major problems for all people in the world. Side effects of chemotherapy and radiation therapy have been known obviously. Since the therapies at present do not provide successful results for some types of cancers, the scientists have long been looking to natural remedies for the treatment of cancer. In this regard, plant-derived compounds were evaluated first because of the findings that use of some plants in some rural populations to treat some types of cancers give rise to positive results. In fact, plants have a long history in the treatment of cancer [1] and studies related to anti-cancer agents from plant sources started in the 1950s with the discovery and development of some alkaloids [2,3].

Since then, much emphasis has been placed on understanding the environmental factors that influence cancer risk. Of these, diet is considered to have a major role. Epidemiological studies have suggested that certain dietary components are associated with lower cancer incidence [4,5]. These include vitamins as well as other phytochemicals, particularly polyphenols. Because phytochemicals are safe at levels found in the diet, they are of interest to those investigating the role of chemoprevention in lowering cancer risk. The polyphenols found in various plants include a wide range of closely related compounds; these include the flavonoids found in tea leaves (catechins), isoflavonoids in soybeans (genistein and daidzein), and stilbenes in red grapes (resveratrol). Each of these has been previously shown to have anticancer properties [6-8]. 
Citation: Zahide Esra Durak, Suleyman Buber, Bahadır Ozturk and Hilmi Kocaoglu (2015) Effects of Aqueous Extracts from Grape and Grape Seed on Adenosine Deaminase Activity in Cancerous and Non cancerous Human Gastric and Colon Tissues. F Nutr Reprt 1(2): 6-9. doi: https:// doi.org/10.24218/fnr.2015.07.

Grape and grape seed is highly enriched in proanthocyanidins. These complex polyphenols have in vitro antioxidant activity [9]; inhibit aromatase enzyme activity [10] and the growth of cancer cells in cell culture [11,12]; prevent or attenuate some diseases including atherosclerosis [13], cataract formation [14], and skin cancer [15]. Grape extract was shown to inhibit the growth of various cancer cells like breast tissue cells in culture $[11,12]$. In fact, grape proanthocyanidins are natural antioxidants which possess a broad spectrum of chemoprotective properties against free radicals and oxidative stress.

Adenosine deaminase (ADA) is an enzyme (EC 3.5.4.4) involved in purine metabolism. It is needed for the breakdown of adenosine and for the turnover of nucleic acids in tissues. ADA is present virtually in all mammalian cells, and it is thought that its primary function in human beings is related to the immune system [16]. However, the full physiological role of ADA is not completely understood [13]. ADA association has also been observed with epithelial cell differentiation, neurotransmission, and gestation maintenance $[13,16,17]$. It has also been proposed that ADA, in addition to its role in adenosine breakdown, stimulates release of excitatory amino acids and is necessary to the coupling of A1 adenosine receptors and heterotrimeric $\mathrm{G}$ proteins $[13,16]$.

As discussed briefly above, although some hypotheses are supposed for the action of grape and its seed in the cancer process, it is possible that in addition to known mechanisms, there should be some others unknown in detail yet. Therefore, further studies are needed. As to the subject, investigation of the effects of aqueous extracts from grape and its seed on ADA activity in cancerous and non cancerous human tissues might give significant results as ADA is a key enzyme in purine nucleotide metabolism and cancer process.

\section{Materials and Methods}

Twenty two cancerous gastric tissues and 22 non-cancerous adjacent gastric tissues were obtained from patients with gastric cancer by surgical operation. Eleven cancer and 11 non cancer colon tissues were similarly obtained from patients with colon cancer. Tissues were first cleaned by saline solution and stored at $-80^{\circ} \mathrm{C}$ until analysis. In the analysis process, they were first homogenized in saline solution $(20 \%$, w/v). After homogenization, samples were centrifuged at $5000 \mathrm{rpm}$ for 30 min to remove debris and to obtain clear supernatant fraction. Analyses were performed in this fraction [18].

The extracts were prepared by soaking ground powder (grape and grape seed) into the distilled water at the concentration of $10 \%$ $(\mathrm{w} / \mathrm{v})$ and waiting for $24 \mathrm{~h}$ at room temperature by continuously rotating. After the debris was removed, supernatants were centrifuged at $10.000 \mathrm{rpm}$ for $20 \mathrm{~min}$ and upper clear part was removed to be used in the assays.

Protein concentrations of the tissues were measured by Lowry method [19] and ADA activity was measured by the method of Guisti [20].

Statistical evaluations were made by using Wilcoxon test and values lower than 0.05 were evaluated significant.

\section{Results}

Results were shown in the Table. First, it should be noted that as to the activity values in the cancerous and non cancerous tissues, no meaningful differences are observed in the present study. However, as to the effect of the extracts, it is clearly seen that grape extract inhibits ADA activity in all the tissues studied. However, grape seed extract inhibits ADA enzyme activity only in the cancerous gastric tissues.

\section{Discussion}

Plants and some nutritional foods are important sources of effective conventional drugs for the treatment of some types of cancers, leading to the development of potential novel agents [21-23]. One of them is grape and its seed, which are known to have some functional constituents with anti cancer potential. Several of the molecules from grape have anticancer potentials on cancer cells. These effects have been observed through in vitro and in vivo animal studies.

Previous experiments showed that grape extracts inhibited growth of breast cancer cells in culture by a mechanism involving cell cycle arrest and ultimately differentiation and nonapoptotic cell death [11]. Additionally, it has been observed that the proanthocyanidins extracted from grape have significant health benefits, as initially suggested [24,25]. In a study, it has been established that concord grape juice added to rat drinking water significantly attenuated tumor multiplicity in the DMBA (Dimethylbenzanthracene) rat model of breast cancer [26], suggesting that the monomeric anthocyanins enriched in grape juice have similar bioactivities as the proanthocyanidinrich grape extract. In a study, it has been shown that topical application of a DMSO (Dimethylsulphoxide)-soluble extract of proanthocyanidin-enriched grape seed extract significantly protected against phorbol ester-induced tumor promotion in a mouse model of skin cancer [14], suggesting that anticancer cell growth activity resided in this oligomeric polyphenol fraction from grape seeds. In addition, the extract oligomers were suggested to be the active agents inhibiting growth of MDAMB468 cells [11]. The mechanism by which dietary composition influences chemopreventive efficacy of grape extracts remains to be determined. Because of the potentially high biomedical value of these bioactivities of the grape phenolic compounds, it is anticipated that future research will address the questions of mechanisms and identification of the bioactive entities from grape juice and seeds.

Therefore, it seems valuable to investigate possible effects of grape and its seed extracts on some critical components having function in the living cells in the body. In this regard, ADA seems of importance since it is a key enzyme in the purine metabolism, inhibition of which may give selective advantage to combat with cancer. Therefore, investigation of possible effects of some natural foods may give useful information about their anticancer potential mechanisms. From a scientific perspective, the use of ADA inhibitors has helped enormously in understanding the mechanism of action of adenosine metabolites and analogs whose catabolism was heretofore neglected with respect to their specificities of action. ADA inhibitors have also enabled us to understand the regulatory processes associated with immunodeficiency characterized by a lack of ADA, and to further understand the maturation of the immune response [27]. Of them, pentostatin is a nucleoside analog that inhibits the activity 
Citation: Zahide Esra Durak, Suleyman Buber, Bahadır Ozturk and Hilmi Kocaoglu (2015) Effects of Aqueous Extracts from Grape and Grape Seed on Adenosine Deaminase Activity in Cancerous and Non cancerous Human Gastric and Colon Tissues. F Nutr Reprt 1(2): 6-9. doi: https:// doi.org/10.24218/fnr.2015.07.

Table: Mean \pm SD values of adenosine deaminase (ADA) enzyme activities (IU/mg protein) in the tissues with and without grape seed (1) and grape (2) extracts.

\begin{tabular}{|l|l|l|}
\hline Groups & A without extract & B with extract-1 \\
\hline I-Non-cancerous colon tissues $(n=11)$ & $5,53 \pm 3,32$ & $6,35 \pm 5,01$ \\
\hline II-Cancerous colon tissues $(n=11)$ & $5,30 \pm 3,46$ & $6,13 \pm 2,54$ \\
\hline III-Non-cancerous gastric tissues $(n=22)$ & $11,19 \pm 9,57$ & $9,24 \pm 7,1$ \\
\hline IV-Cancerous gastric tissues $(n=22)$ & $11,45 \pm 8,57$ & $4,81 \pm 3,48 *$ \\
\hline
\end{tabular}

All the data are presented as means \pm SD

* $\mathrm{P}<0.05$ between samples treated with and those without extracts as analyzed by wilcoxon test.

Extract-1: Grape seed extract

Extract-2: Grape extract

of the enzyme adenosine deaminase. Inhibition of adenosine deaminase blocks the deamination of adenosine to inosine, and deoxyadenosine to deoxyinosine in the purine salvage pathway. This accumulation of metabolites inhibits ribonucleotide reductase, which depletes the nucleotide pool and limits DNA synthesis [28].

Looking at our results, it seems that there are no differences between ADA activities of cancerous and non cancerous tissues for both tissues as well. In some previous studies, higher ADA activities were however reported in several types of cancer tissues including gastric cancer $[29,30]$. The differences observed between studies might be resulted from the fact that patients were under different chemotherapy protocols before surgical operation. For example, in our study all of the patients had previously received intense different chemotherapies before surgical operation. These kinds of therapies might decrease ADA enzyme activity in cancerous tissue.

Furthermore, our results suggest that constituents in aqueous grape extract inhibit ADA activity in all the tissues studied. However, seed extract can inhibit the enzyme only in the cancerous gastric tissue. This difference between grape and its seed extracts shows that structural constituents having potential to inhibit ADA enzyme are different in grape and its seed. It seems quite possible that in addition to other proposed mechanisms, accumulated adenosine due to the inhibition of ADA enzyme might play an important function in the anti-cancer properties of grape, possibly through inhibition of ribonucleotide reductase and depletion of nucleotide pool for new DNA synthesis. However, subject needs further studies including in vivo animal studies to obtain more data on the possible anticancer potentials of grape and its seed constituents.

\section{References}

1. Cragg GM, NewmanDJ. Plants as a source of anti-cancer agents. J Ethnopharmacol. 2005; 100(1-2): 72-9.

2. Noble RL. The discovery of the vinca alkaloids-chemotherapeutic agents against cancer. Biochem Cell Biol. 1990; 68(12): 1344-51.

3. HimesRH. Interactions of the catharanthus (Vinca) alkaloids with tubulin and microtubules. Pharmacol Ther. 1991; 51(2): 257-67.

4. DollR. The epidemiology of cancer. Cancer. 1980; 45(10): 2475-85.

5. GerberM. The comprehensive approach to diet: a critical review. J Nutr. 2001; 131(11 Suppl): 3051-5.

6. Park OJ, Surh YJ. Chemopreventive potential of epigallocatechin gallate and genistein: evidence from epidemiological and laboratory studies. Toxicol Lett. 2004; 150(1): 43-56.
7. BarnesS, PetersonTG. Biochemical targets of the isoflavone genistein in tumor cell lines. Proceedings of the Society for Experimental Biology and Medicine. Society for Experimental Biology and Medicine (New York, N.Y.). 1995; 208(1): 103-8.

8. Mgbonyebi OP, Russo J, Russo IH. Antiproliferative effect of synthetic resveratrol on human breast epithelial cells. Int J Oncol. 1998; 12(4): 865-9.

9. Yamaguchi F, Yoshimura Y, Nakazawa H, Ariga T. Free radical scavenging activity of grape seed extract and antioxidants by electron spin resonance spectrometry in an $\mathrm{H}(2) \mathrm{O}(2) / \mathrm{NaOH} / \mathrm{DMSO}$ system. J Agric Food Chem. 1999; 47(7): 2544-8.

10. Eng ET, Ye J, Williams D, Phung S, Moore RE, Young MK, et al. Suppression of estrogen biosynthesis by procyanidin dimers in red wine and grape seeds. Cancer Res. 2003; 63(23): 8516-22.

11. Agarwal C, Sharma Y, Zhao J, Agarwal RA.Polyphenolic fraction from grape seeds causes irreversible growth inhibition of breast carcinoma MDA-MB468 cells by inhibiting mitogen-activated protein kinases activation and inducing G1 arrest and differentiation. Clin Cancer Res. 2000; 6(7): 2921-30.

12. Sharma G, Tyagi AK, Singh RP, Chan DC, AgarwalR. Synergistic anticancer effects of grape seed extract and conventional cytotoxic agent doxorubicin against human breast carcinoma cells. Breast Cancer Res Treat. 2004; 85(1): 1-12.

13. Wilson DK, Rudolph FB, Quiocho FA. Atomic structure of adenosine deaminase complexed with a transition-state analog: understanding catalysis and immunodeficiency mutations. Science. 1991;252(5010): 1278-84.

14. YamakoshiJ, SaitoM, KataokaS, TokutakeS. Procyanidin-rich extract from grape seeds prevents cataract formation in hereditary cataractous (ICR/f) rats. J Agric Food Chem. 2002; 50(17): 4983-8.

15. BomserJA, SingletaryKW, Wallig MA, Smith MA. Inhibition of TPA-induced tumor promotion in CD-1 mouse epidermis by a polyphenolic fraction from grape seeds. Cancer Lett. 1999; 135(2): 151-7.

16. Cristalli G, CostanziS, LambertucciC, LupidiG, VittoriS, VolpiniR, et al. Adenosine deaminase: functional implications and different classes of inhibitors. Med Res Rev. 2001; 21(2): 105-28.

17. AghaeiM,Karami-Tehrani F, Salami S, Atri M. Adenosine deaminase activity in the serum and malignant tumors of breast cancer: the assessment of isoenzyme ADA1 and ADA2 activities. Clin Biochem. 2005; 38(10): 887-91.

18. Durak I,BiriH, Ergüderi,Devrim E, Şenocak Ç, Avcı A. Effects of garlic and black grape extracts on the activity of adenosine deaminase from cancerous and noncancerous human urinary bladder tissues. Medicinal Chemistry Research. 2007; 16(6): 259-65. doi: 10.1007/ s00044-007-9027-6. 
Citation: Zahide Esra Durak, Suleyman Buber, Bahadır Ozturk and Hilmi Kocaoglu (2015) Effects of Aqueous Extracts from Grape and Grape Seed on Adenosine Deaminase Activity in Cancerous and Non cancerous Human Gastric and Colon Tissues. F Nutr Reprt 1(2): 6-9. doi: https:// doi.org/10.24218/fnr.2015.07.

19. LowryOH,RosebroughNJ, FarrAL, RandallRJ. Protein measurement with the Folin phenol reagent. J Biol Chem. 1951; 193(1): 265-75.

20. GuistiG. Enzyme activities. Methods of enzymatic analysis. Weinheim Bergest: Verlag chemia; 1974. 1087-91 p.

21. Chang CJ, Ashendel CL, GeahlenRL,McLaughlin JL, WatersJ. Oncogene signal transduction inhibitors from medicinal plants. In Vivo. 1996; 10(2): 185-90.

22. Desai AG,Qazi GN,Ganju RK,El-Tamer M, Singh, J, Saxena AK, et al. Medicinal plants and cancer chemoprevention. Curr Drug Metab. 2008; 9(7): 581-91.

23. Lee HZ, Hsu SL, Liu MC, Wu CH. Effects and mechanisms of aloeemodin on cell death in human lung squamous cell carcinoma. Eur J Pharmacol. 2001; 431(3):287-95.

24. Masquelier J, MichaudJ, Laparra J, DumonM. Flavonoids and pycnogenols. International journal for vitamin and nutrition research. Internationale Zeitschrift für Vitamin-und Ernährungsforschung. Journal international de vitaminologie et de nutrition. 1979; 49(3):307.

25. Murray M, PizzornoJ. Procyanidolic oligomers. The Textbook of Natural Medicine. 2nd ed. London: Churchill Livingston; 1999. 899$902 \mathrm{p}$.
26. Singletary KW, Stansbury MJ, Giusti M, Van Breemen RB, Wallig M, Rimando A. Inhibition of rat mammary tumorigenesis by concord grape juice constituents. J Agric Food Chem. 2003; 51(25):7280-6.

27. Glazer RI. Adenosine deaminase inhibitors: their role in chemotherapy and immunosuppression. Cancer Chemother Pharmacol. 1980; 4(4):227-35. doi: 10.1007/BF00255266.

28. Brown JB, LeeG, Grimm, GR, Barrett TA. Therapeutic benefit of pentostatin in severe IL-10-/- colitis. Inflamm Bowel Dis. 2008; 14(7):880-7. doi: 10.1002/ibd.20410.

29. Durak I, Perk H, KavutçuM, CanbolatO, AkyolÖ, BedükY. Adenosine deaminase, 5' nucleotidase, xanthine oxidase, superoxide dismutase, and catalase activities in cancerous and noncancerous human bladder tissues. Free Radic Biol Med. 1994; 16(6):825-31.

30. Durakl, CetinR, CanbolatO, CetinD, YurtarslaniZ, ÜnalA. Adenosine deaminase, 5'-nucleotidase, guanase and cytidine deaminase activities in gastric tissues from patients with gastric cancer. Cancer Lett. 1994; 84(2):199-202. 